\title{
A Distributed Signaling Fast Mobile IPv6 Scheme for Next Generation Heterogeneous IP Networks
}

\author{
Mohtasim Abbassi, Shahbaz Khan, and M. Rahman \\ Department of Telecommunication Engineering, \\ University of Engineering \& Technology, \\ Peshawar (Mardan Campus), 23200, Pakistan \\ mohtasimabbassiayahoo.com
}

\begin{abstract}
The Next-Generation Wireless Networks (NGWNs) are believed to exhibit a heterogeneous environment which imposes various challenges, among them Mobility Management is most crucial. The FMIPv6 protocol has been standardized by IETF to address certain shortcomings of the baseline Mobile IPv6. However, it does not directly support vertical handovers. Therefore, research is still going on to develop more effective IPv6 based vertical handoff protocols for efficient support of frequent and seamless roaming. This paper presents a new protocol named Distributed Signaling Fast Mobile IPv6 (DS-FMIPv6) which effectively addresses reduction of handoff latency, signaling costs and power overheads for a better support of seamless \& frequent vertical handoffs.
\end{abstract}

Keywords: Mobility Management, NGWNs, FMIPv6, DS-FMIPv6.

\section{Introduction}

The Next-Generation wireless networks are extensively recommended to be all-IPbased. Therefore, the IPv6-based Mobility Management protocols are of significant importance. The FMIPv6 [1] proposed by IETF aims towards improving the performance of the fundamental MIPv6 protocol [2]. These protocols are however hindered by several drawbacks of which the handoff latency is most important. Moreover, in case of frequent handovers, signaling and power overheads incurred by these schemes become even more critical. These protocols also require improvements to become suitable enough to efficiently fulfill several stringent requirements like context transfers during vertical handoffs.

In this paper, a vertical handoff protocol named Distributed Signaling FMIPv6 (DS-FMIPv6) is presented. The signaling involved in this handoff protocol is distributed among MN, Current Access Router (CAR) and the New Access Router (NAR) hence the scheme is named as Distributed Signaling FMIPv6 scheme. The performance of FMIPv6 is further improved in DS-FMIPv6 by carrying out selected signaling procedures in advance i.e. prior to the start of actual handover and by increasing the functionality of routers. The protocol operation relies on a network selection algorithm evaluated at the Current Access Router (CAR) which evaluates the best 
suitable network beforehand for the current $\mathrm{MN}$ status. This algorithm is assumed to encompass all necessary parameters required for network selection. Detailed analysis shows that DS-FMIPv6 scheme significantly reduces the handoff latency along with signaling and power overheads when an MN undergoes frequent handovers.

The rest of paper is organized as follows: in section 2, an overview of IETFproposed FMIPv6 protocol along with its few extensions is presented. Section 3 presents the proposed DS-FMIPv6 protocol. Performance analysis of DS-FMIPv6 is explained in Section 4. Finally, section 5 concludes the paper and sets directions for future research in connection with this topic.

\section{Fast Mobile IPv6 and Its Extensions}

\subsection{Fast Mobile IPv6}

FMIPv6 is proposed to reduce handoff delay and to minimize the services disruption incurred with MIPv6 during handoffs. The most invigorating feature of FMIPv6 is that it relies on link layer information (L2 Trigger) to predict or to rapidly respond to handoff events (Predictive and Reactive mode respectively). When an MN detects its movement towards NAR, by using L2 Trigger, it exchanges Router Solicitation for Proxy Advertisement (RtSolPr) and Proxy Router Advertisement (PrRtAdv) messages with Previous Access Router (PAR) to configure a new Care-of Address (nCoA). The $\mathrm{MN}$ sends FBU to PAR in order to associate its previous CoA with New CoA. In order to prevent routing failure, a bidirectional tunnel between PAR and NAR is established by using Handover Initiate (HI) and Handover Acknowledgment (HAck) messages. On communicating FBU to PAR, the MN undergoes L2 handoff procedures. After successful L2 Handoff the MN announces its presence on new link by using Unsolicited Neighbour Advertisement (UNA) message. The NAR at this stage starts delivering packets to the MN. The Binding Updates to HA \& CN are carried out in similar fashion as for MIPv6.

\subsection{Enhancements in Fast Mobile IPv6}

Various enhancements in FMIPv6 are proposed with different aims and objectives, but their efficiency is hindered by certain limitations. An overview of few schemes is presented in this sub-section. In [3], the handoff latency of FMIPv6 is improved by making certain fundamental enhancements in various processes involved. The movement detection \& tunnel establishment procedures are merged, resulting in reduction of latency because of DAD procedure. The Binding Update to the HA/CN is brought forward to the time before the layer 2 handover which further improves the latency problem. In [4], an Intelligent FMIPv6 (iFMIPv6) scheme is designed specifically for WLANs. This scheme addresses the reduction in Scanning Delay, where the Access Point will have Pre-knowledge of its neighbourhood and the MN is intelligent enough knowing its mobility pattern and thus the next stop of its movement. Therefore, at the time of handoff, the MN only needs to scan the selected AP by probing the corresponding operating channel, thus reducing the overall scanning delay. In [5], an 
efficient handover scheme between WLAN and 3G-UMTS is proposed utilizing Pre-Authentication in order to achieve a lossless vertical handover. An efficient handoff decision algorithm provides three link layer triggers for setting off the MN to detect the movement to a new subnet, to start Pre-Authentication, and to initiate Fast Binding Update. This Pre-Authentication concept reduces the overall service disruption time to a reasonable level. In [6], FMIPv6 based cross layer optimized vertical handover scheme is proposed for Mobile WiMAX, UMTS and CDMA networks. In this scheme, certain Layer 2 and Layer 3 messages are reordered or combined to eliminate redundant signaling. In [7], pseudo-binding FMIPv6 (pFMIPv6) scheme is presented which addresses the fast moving issue of the MN. The Access Router informs mobile node about the existence of other networks around the current network. The $\mathrm{MN}$ in turn creates several CoAs and initiates pseudo Fast Binding Update (pFBU) with all its neighbouring networks, even though it does not detect the signal of the next network yet. In this way, time deficiency involving these $\mathrm{HO}$ procedures for a fast moving $\mathrm{MN}$ is reduced to a high extent.

\section{The DS-FMIPv6 Protocol}

The signaling involved in DS-FMIPv6 is majorly derived from the MIPv6 and FMIPv6. The FBAck message is omitted and a new message Binding Update Initiate (BUI) is defined for its efficient protocol operation. Following steps constitute the protocol operation of DS-FMIPv6

i. Any change in status of MN (e.g. its average velocity, direction or imminent active application) is reported to the CAR through an enhanced RtSolPr (eRtSolPr) message. The RtSolPr message in FMIPv6 is enhanced to form eRtSolPr by defining a new option field which carries information about the above defined parameters. The Link Local Address (LLA) of MN is also communicated using LLA option.

ii. The CAR evaluates the handoff decision algorithm (HDA) using MN's information from eRtSolPr message and neighbouring networks information from the Router's cache. The prospective new network of MN is evaluated and new Care-of Address (nCoA) is formulated using LLA and network prefix. [8]

iii. The CAR now initiates the Duplicate Address Detection (DAD) to test the uniqueness of the CoA, by exchanging pHI (pseudo HI) and pHAck (pseudo HAck) messages with NAR. This exchange of messages also establishes a tunnel between CAR and NAR, which initially remains inactivated. The establishment of tunnel beforehand can be more effective in reduction of packet loss during handovers.

iv. CAR sends ePrRtAdv (enhanced PrRtAdv) message to inform MN its most suitable network by communicating its new CoA to it. 


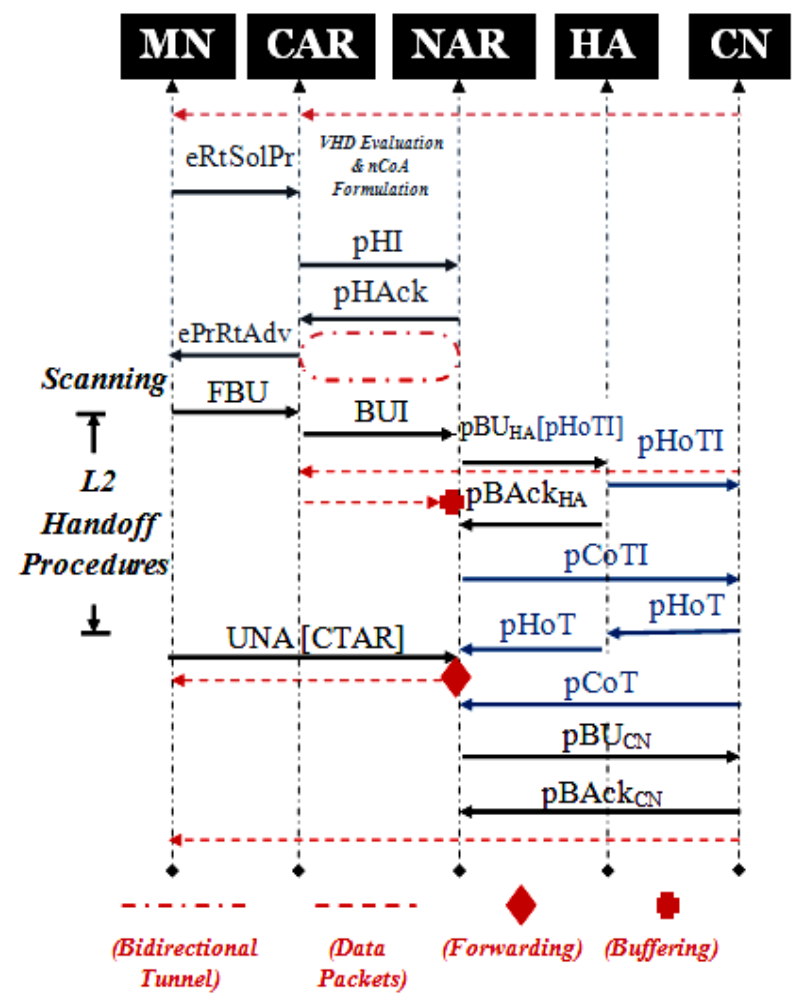

Fig. 1. Signaling Sequence of DS-FMIPv6 Scheme

v. Acquiring nCoA, the $\mathrm{MN}$ sends FBU message to inform CAR that it is ready to handoff to new network. The CAR thus discontinues forwarding traffic to it and starts forwarding packets to NAR through already established tunnel. The CAR also sends a Binding Update Initiate (BUI) message to NAR piggybacking CTD (Context Transfer Data) [9] message in order to inform it that a new $\mathrm{MN}$ is about to join it, and instructs it to initiate the Binding Update procedure with CN/HA.

vi. The MN, on the other hand, scans new network and initiates Layer 2 (L2) handoff. It then announces its presence on the NAR through UNA message. CTAR (Context Transfer Activate Request) message is also piggybacked on it, which carries an authorization token to ascertain the reliability of the Context Transfer. At this instant the protocol operation involving MN completes and $\mathrm{MN}$ is ready to receive data packets from NAR routed through CAR.

vii. The Binding Update procedure on NAR begins when the pHoTI message piggybacked on $\mathrm{pBU}_{\mathrm{HA}}$ (pseudo- $\mathrm{BU}_{\mathrm{HA}}$ ) is sent to HA. This allows the simultaneous initiation of $\mathrm{pBU}$ procedure to $\mathrm{HA}$ and Return Routability (RR). The HA then forwards pHoTI to $\mathrm{CN}(\mathrm{s})$, and responds the NAR by $\mathrm{pA}_{\mathrm{HA}}$ (pseudo-BA $\mathrm{HA}_{\mathrm{H}}$ ) message. 
viii. The NAR also sends the pCoTI (pseudo CoTI) message to the CN. The response of pHoTI and pCoTI message from $\mathrm{CN}$ is pHoT and pCoT messages respectively. The pHoT message is routed to NAR via HA. The RR procedure completes at this stage.

ix. After completion of RR, the NAR can now send the pseudo-Binding Update to Correspondent Node $\left(\mathrm{pBU}_{\mathrm{CN}}\right.$ ). The $\mathrm{CN}$ in turn responds with a $\mathrm{pBA}_{\mathrm{CN}}$ (pseudo$\mathrm{BU}_{\mathrm{CN}}$ ) message to NAR. At this instant the NAR will start receiving data traffic directly from CN(s). This completes the DS-FMIPv6 protocol operation. The signaling flow between different entities is shown in the Figure 1.

\section{Performance Analysis}

\subsection{Handoff Latency}

The Handoff Latency is referred to as the time interval from the moment that packets cannot be sent or received to the moment that the mobile node can communicate directly with $\mathrm{CN}$ via new Access Router [3]. The overall handoff delay for FMIPv6 and DS-FMIPv6 are given as;

$$
\begin{gathered}
T_{F M I P v 6}=T_{D A D}+T_{F B A c k}+T_{L 2}+T_{U N A}+T_{B U} \\
T_{D S-F M I P v 6}=\max \left\{T_{L 2}+T_{U N A}+T_{\text {arrival }}, T_{B U I}+T_{p B U}\right\}
\end{gathered}
$$

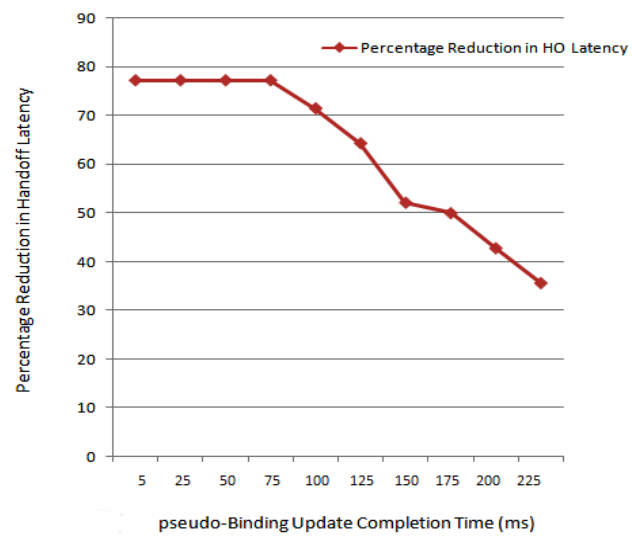

Fig. 2. Percentage Reduction of Handoff Latency in DS-FMIPv6 in comparison to FMIPv6

\subsection{Signaling Cost}

The Advanced DAD procedure [10] incorporated in DS-FMIPv6 eliminates the probability of any Reactive Mode. Here for comparison, we will assume successful anticipation of handover and will compare the signaling of predictive mode of FMIPv6 with DS-FMIPv6. 


$$
\begin{aligned}
& C_{F M I P v 6}=4 C_{M N, P A R}+3 C_{P A R, N A R}+2 C_{M N, N A R}+5 P C_{A R}+C_{B U}+C_{R R} \\
& C_{D S-F M I P v 6}=3 C_{M N, C A R}+4 C_{C A R, N A R}+2 C_{M N, N A R}+6 P C_{A R}+C_{p B U}
\end{aligned}
$$
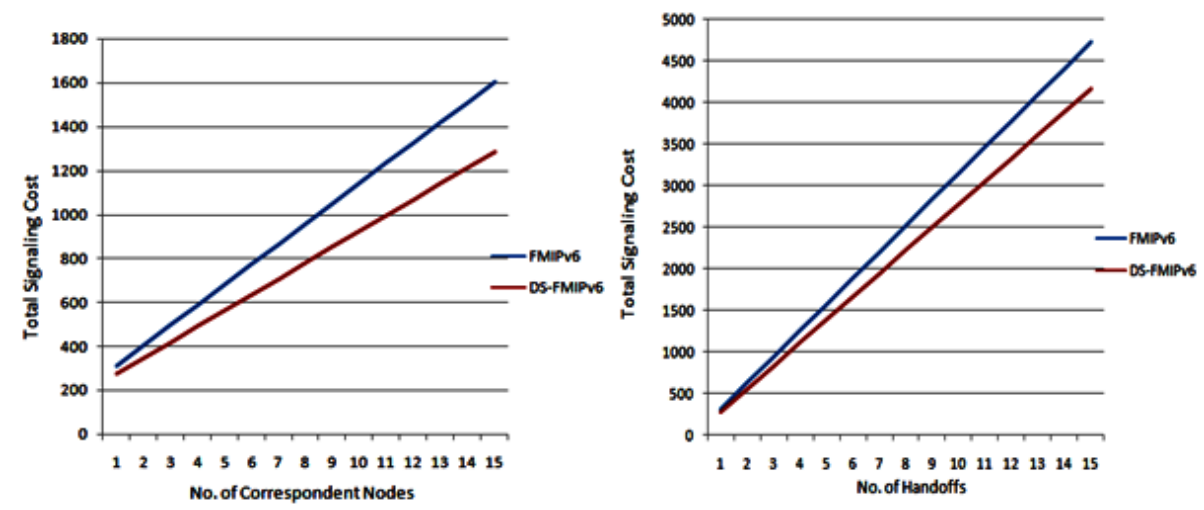

Fig. 3. Signaling Cost comparison of FMIPv6 and DS-FMIPv6

\subsection{Power Consumption}

Evaluation of a supportive handoff decision algorithm at Access Router in DSFMIPv6 facilitates MN to turn on only the suitable network interface at the time of handoff. Traditionally, there can be three other possibilities for interface activation of $\mathrm{MN}$ i.e. all interfaces active all the time, active periodically, or at the time of handoff. The power consumption for each of these cases denoted by $\mathrm{P}_{\mathrm{D}}, \mathrm{P}_{\mathrm{A}}, \mathrm{P}_{\mathrm{P}}$ and $\mathrm{P}_{\mathrm{H}}$ respectively is mathematically formulated as;

$$
\begin{gathered}
\mathrm{P}_{\mathrm{A}}=\mathrm{P}_{\mathrm{S}}+\sum_{\mathrm{n}_{\mathrm{h}}} \mathrm{t}_{s} \cdot \sum_{\mathrm{N}-1} \mathrm{P}_{\mathrm{IDLE}}+\mathrm{n}_{\mathrm{h}} \cdot \mathrm{t}_{\mathrm{HO}} \cdot \sum_{1}^{\mathrm{N}} \mathrm{P}_{\mathrm{HO}} \\
\mathrm{P}_{\mathrm{p}}=\mathrm{P}_{\mathrm{S}}+\frac{0.5}{3}\left(\sum_{\mathrm{n}_{\mathrm{h}}} \mathrm{t}_{\mathrm{s}} \cdot \sum_{\mathrm{N}-1} \mathrm{P}_{\mathrm{IDLE}}\right)+\mathrm{n} \cdot \mathrm{t}_{\mathrm{HO}} \cdot \sum_{\mathrm{N}} \mathrm{P}_{\mathrm{HO}} \\
\mathrm{P}_{\mathrm{H}}=\mathrm{P}_{\mathrm{S}}+\mathrm{n}_{\mathrm{h}} \cdot \mathrm{t}_{\mathrm{HO}} \cdot \sum_{\mathrm{N}} \mathrm{P}_{\mathrm{HO}} \\
\mathrm{P}_{\mathrm{D}}=\mathrm{P}_{\mathrm{S}}+\mathrm{n}_{\mathrm{h}} \cdot \mathrm{t}_{\mathrm{HO}} \cdot \mathrm{P}_{\mathrm{HO}}
\end{gathered}
$$

For comparison, we consider an $\mathrm{MN}$ undergoing 15 handoffs among four networks with different power consumptions in each state $[11,12]$. Results show that interface management in DS-FMIPv6 incurs the lowest possible power consumption. 
Table 1. Example Scenario for Power Consumption comparison

\begin{tabular}{|lcccccccc|}
\hline Interface State & ID & UL & DL & UL & ID & UL & DL & DL \\
Network & GSM & WLAN & UMTS & GSM & UMTS & WLAN & CDMA & WLAN \\
ts(s) & 45 & 20 & 70 & 20 & 35 & 120 & 80 & 90 \\
Interface State & UL & ID & DL & DL & UL & DL & ID & DL \\
Network & UMTS & WLAN & GSM & CDMA & UMTS & WLAN & GSM & CDMA \\
ts(s) $^{(\mathbf{s})}$ & 60 & 30 & 140 & 80 & 40 & 100 & 30 & 40 \\
\hline
\end{tabular}

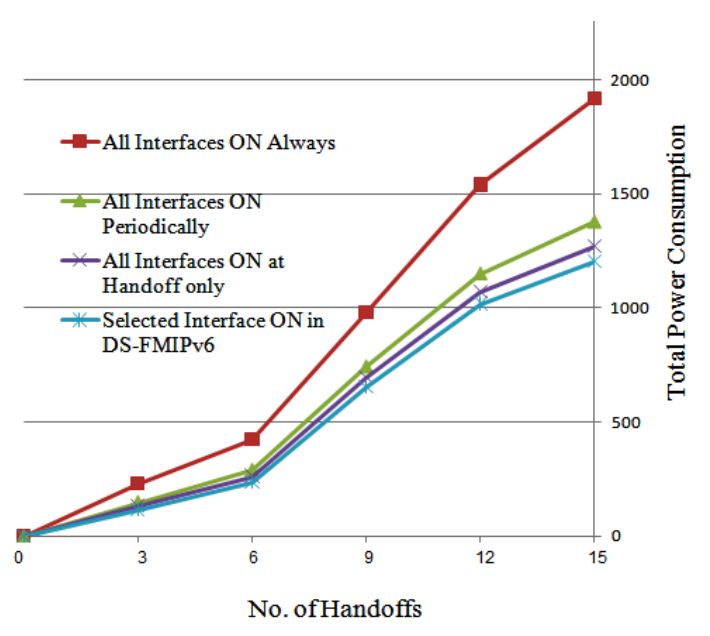

Fig. 4. Power Reduction by DS-FMIPv6 Interface Management

Table 2. System Parameters

\begin{tabular}{|lll|}
\hline Parameter & Notation & Value \\
\hline (a). Handoff Latency & & \\
\hline Layer 2 Handoff Delay & $\mathrm{T}_{\mathrm{L} 2}$ & $50 \mathrm{~ms}$ \\
Message Processing Delay at any Node X & $\mathrm{T}_{\mathrm{X}}(\mathrm{X})$ & $5 \mathrm{~ms}$ \\
Link Delay (among Local Nodes A \& B) & $\mathrm{T}_{\mathrm{X}}(\mathrm{A}, \mathrm{B})$ & $10 \mathrm{~ms}$ \\
Link Delay (among Remote Nodes like HA/CN) & $\mathrm{T}_{\mathrm{X}}(\mathrm{A}, \mathrm{HA} / \mathrm{CN})$ & $15 \mathrm{~ms}$ \\
\hline (b). Signaling Cost & & \\
Signaling Cost between MN \& PAR/CAR & $\mathrm{C}_{\mathrm{MN}, \mathrm{PAR} \text { CAR }}$ & 5 \\
Signaling Cost between PAR/CAR \& NAR & $\mathrm{C}_{\mathrm{PAR} / \mathrm{CAR}, \mathrm{NAR}}$ & 7 \\
Processing Cost at any AR & $\mathrm{PC}_{\mathrm{xAR}}$ & 8 \\
Signaling Cost between MN \& HA & $\mathrm{C}_{\mathrm{MN}, \mathrm{HA}}$ & 15 \\
Signaling Cost between any AR \& HA & $\mathrm{C}_{\mathrm{xAR}, \mathrm{HA}}$ & 10 \\
Signaling Cost between MN \& CN & $\mathrm{C}_{\mathrm{MN}, \mathrm{CN}}$ & 15 \\
Processing Cost at HA & $\mathrm{PC}_{\mathrm{HA}}$ & 24 \\
Processing Cost at CN & $\mathrm{PC}_{\mathrm{CN}}$ & 4 \\
Signaling Cost between HA \& CN & $\mathrm{C}_{\mathrm{HA}, \mathrm{CN}}$ & 10 \\
\hline (c). Power Consumption & & \\
Power consumption per second in State "x" & $\mathrm{P}_{\mathrm{x}}$ & Manifold (Table 3) \\
Session time for MN in State " $\mathrm{x}$ & $\mathrm{t}_{\mathrm{x}}$ & Manifold (Table 3) \\
Interface Activation time for Handoff & $\mathrm{t}_{\mathrm{H} \mathrm{O}}$ & 1 sec \\
\hline
\end{tabular}


Table 3. Partial Expressions

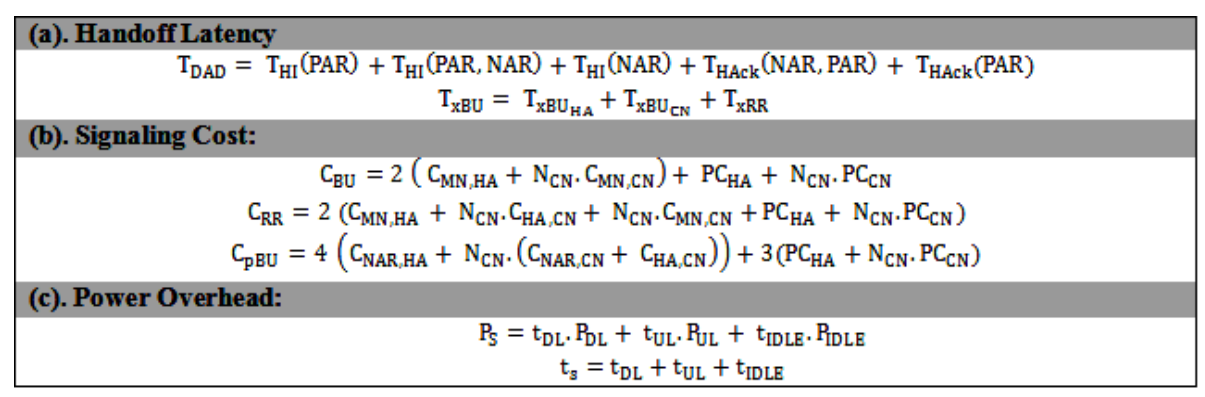

\section{Conclusion}

The IETF solutions proposed for Mobility Management are not compact enough to support vertical handoffs. Also their handoff delays adversely affect delay sensitive and real-time applications. Other issues of signaling Cost and Power consumption at MNs become equally crucial in case of recurring handoffs. In this paper, a novel DSFMIPv6 protocol is proposed which effectively reduces the handoff latency imposed by FMIPv6 from 35-75\%. The proposed scheme also promises the lowest possible signaling cost \& power consumption of MNs. In future, the obtained numerical results will be validated through simulations by modeling the protocol on a suitable simulator in order to provide a better analysis of these metrics.

\section{References}

1. Koodli, G.: Fast Handover for Mobile IPv6, IETF RFC 5568

2. Johnson, D.B., Perkins, C.E., Arkka, J.: Mobility Support in IPv6, IETF RFC 3775

3. Li, R., Li, J., Wu, K., Xiao, Y., Xie, J.: An Enhanced Fast Handover with low latency for Mobile IPv6. IEEE Transactions on Wireless Communications 7(1) (January 2008)

4. Zhang, L.J., Zhang, L., Pierre, S.: Intelligent Fast Handover Scheme for Mobile IPv6based Wireless Local Area Networks. IJCSNS 9(8) (August 2009)

5. Menezes, S., Venkatesan, S., Rho, K.H.: An Efficient Handover Scheme based on Fast Mobile IPv6. In: IEEE 64th VTC-2006 Fall, pp. 1-5 (September 2006)

6. Jo, J., Cho, J.: A Cross-Layer Vertical Handover between Mobile WiMAX and 3G Networks. In: IWCMC 2008, pp. 644-649 (August 2008)

7. Kim, H.: An Enhancement of FMIPv6 for Packet Radio Networks which Supports the QoS Provisioning on the MIPv6. JDCTA 3(2) (June 2009)

8. Thomson, S., Narten, T., Jinemei, T.: IPv6 Stateless Address Autoconfiguration. IETF RFC 4862 (September 2007)

9. Loughney, J. (ed.), Nakhjiri, M., Perkins, C., Koodli, R.: Context Transfer Protocol (CXTP). IETF RFC 4067 (July 2005) 
10. T-Trong, S., Tursunova, S., Kim, Y.-T.: Enhanced Vertical Handover in Mobile IPv6 with Media Independent Handover Services and Advance Duplicate Address Detection. In: KNOM Conference (2008)

11. Salawu, N., Onwuka, E.N.: Energy Optimization Mechanism for Mobile Terminals using Vertical Handoff between WLAN and CDMA2000 Networks. Leonardo Electronic Journal and Practices and Technologies (15), 51-58 (July-December 2009)

12. Perrucci, G.P., Fitzek, F.H.P., Sasso, G., Kellerer, W., Widmer, J.: On the Impact of 2G and 3G Network Usage for Mobile Phones' Battery Life. In: EW 2009, pp. 255-259 (December 2009) 